\title{
The Burst of the Latent: Politics of mobile connectivity
}

\author{
Hassan Choubassi \\ Lebanese International University \\ Fine Arts \& Design Department \\ Lebanese International University \\ Michel Abi Chahla street, Mousytbeh \\ Beirut-Lebanon \\ hassan.choubassi@liu.edu.lb
}

\begin{abstract}
In virtual reality the body is doomed to immobility, though it is connected to the wonderful world of the screen but it only means a static connectivity, an imprisonment of the physical body behind the screen. The advent of mobile connectivity marked a revolution in communication technology. With the new technologies of smart phones, the image has taken on a new dimension and the space changed from being an enclosed, cocooned space to the actual space of the real. The image that was latent in the virtual and trapped within the boundaries of the catatonic digital stationary of a desktop computer is now loose at large.
\end{abstract}

The flux is so fast and omnipresent that the boundaries between the two entities of the virtual and the actual are blurry and inconsistent to the extent that the individual user of media augmentation cannot distinguish the difference. The latent image of the virtual exploded in the actual augmenting it to become an actual and physical space of hyperactivity and virtual flux. Manipulation of the image is becoming manipulation of life itself and thus the creation of an augmented space in the actual becomes a prerequisite.

Augmented reality. Virtual Reality. Smart phones. Mobile connectivity. Teletopia.

\section{INTRODUCTION}

Living a situation of excess of information would unavoidably lead to explosion, explosion of information in that context, and because this information is instantaneous, continuous and interactive, it tends to be unstable and ambiguous, and when mobility is added, it becomes a recipe for explosion. This is what Paul Virilio calls the "information bomb"(Virilio, 2005) making an analogy with the atom bomb: he goes on to say: "After the first bomb, the atom bomb, which was capable of using the energy of radioactivity to smash matter, the specter of a second bomb is looming at the end of this millennium. This is the information bomb, capable of using the interactivity of information to wreck the peace between nations."1 For him, information can be much more dangerous then nuclear weapons as it is a pivotal factor in any modern society and it is embedded in the infrastructure as well as in the superstructure of any nation. Although Virilio did not specify the exact kind of explosion that this information bomb will cause but his predictions were realized on so many levels, the explosion came as an economical crisis, as political instability and more importantly in the street in the form of a wave of civil demonstrations in all major cities around the world, and in the form of an explosion of demonstrations against totalitarian regimes in most of the Arab countries in what is called the "Arab spring".

In a lecture performance entitled: "The Pixelated Revolution"(Mroue, 2011) in 2011 Rabih Mroue exemplified this excess of images in the instantaneous reporting of the Arab revolutions and how those images 
where capturing the moments of death or the death of the moment, his work focused on the Syrian revolution were he collected videos and photos directly podcasted on the internet and he analysed them in a more or less anthropological manner focusing on the immediacy and the "truth" of the image that overshadows the low quality and pixilation of that image, moreover for him, it is this exact status of low resolution that makes this image a true reporter that gave it credibility and thus its aggressive nature, a violent image of the real as it is, for him the "Syrians are filming their own death". Mroue's work studies the image that is generated by smart phones or hand-held mobile devices and how it encapsulate a true insight into the savage reality of the Syrian conflict, while doing that he also studies the implication of social media in sharing and propagating the real image of violence without manipulation or censorship. This performance looks at the Syrian dilemma from a radical non-mediated perspective, as Rabih puts it: "an outlook of the revolution form the view point of those who are 'now-and-here', and its interpretation by those who are 'now-but-there' witnessing the events instantaneously and continuously from afar."

For Jean Baudrillard the excess of information is also a very unstable material that should be handled with care, the surplus of information becomes likes poison, it intoxicate and would transform a scene into obscene, transform signification into banality and renders the production of images into a pornographic act of informing the disenchanted. He goes on to say that: "The result is therefore not at all any additional information or any light on reality, but on the contrary, because of the fact that we will never in the future be able to separate reality from its statistical, simulative projection in the media, a state of suspense and of definitive uncertainty about reality."2 New technologies Information are produced by the masses for the masses, for Baudrillard this is self cannibalism the infatuation of the social with its own image and the repetition of this image in an infinity of variations becomes like eating oneself. History itself is nullified with this excess of information that is itself an excess of eventfulness of history, in that sense historical action is cancelled with the negation of the notion of time. When the speed of information goes along, or even surpasses, the actual event that it is supposed to report; information itself becomes history. When the image becomes instantaneous time becomes zero and thus space-time under such conditions becomes virtual space and zero time cancelling with it the notion of history and thereof the signified in the semiotics sense or actual reality itself.

All this pessimism about the media and about information that Virilio and Baudrillard expressed were only on the level of the virtual, the image, for them, did not yet take its new dimension of mobile interactivity. What is intriguing now is the realization that what they were talking about was only the tip of the iceberg; the best was yet to come and the real obscenity will reach its peak with the advent of mobile connectivity and the wide use of those technologies by the masses. Mobile phones are in constant proximity to the user's body, bodies are becoming cyborgs as they are more and more depending on smart phones in daily life. Not only instantaneous and continuous, but information also becomes mobile, retrieving and providing repetitive information now is possible on the move, in actual reality in the street, right in the middle of this "desert of the real itself" that Baudrillard thought will never be inhabited again. The explosion is now taking place in front of all those cameras of the mobile phones that are reporting it in slow motion and broadcasting it on the spot as if from a scene of a Hollywood action movie. The virtual image was not obscene like Baudrillard claimed; the obscenity of the image is now and here with this mixed augmentation that is happening instantaneously in an actual space and real time but overlapped with virtual parameters of the non-space and differed time, a combination that is short-circuiting the process of perception. While exposed to the real and the virtual together on the same stage one cannot but speculate the differences between the two realms, the similarities are mesmerizing and the experience of a full $3 \mathrm{D}$ emersion becomes an explosion of fantasies and desires.

\section{FROM IMPLOSION TO EXPLOSION}

This excess of information is produced instantaneously by a society who is watching live what it is streaming and directly interacting with it. A self-intoxicating image, a latent catastrophe in the virtual that had recently burst into explosion when this producer of the image is also on the move in physical actuality as the new technologies of augmentation allows mobile connectivity. She/he is no more cocooned in her/his stationary post isolated from her/his direct surrounding but on the contrary, she/he is in proximity with her/his physical environment interacting with her/his surrounding and at the same time connected to the world of virtuality and interacting with another layer of another reality at the same time. Moreover, she/he has the ability to combine those two realities together. The user of the technologies of mobile connectivity is able to directly share the physical experience of the actual with other users in the realm of the virtual that, in their turn are also sharing their experiences in their own actuality. The image that was latent in the virtual and trapped within the boundaries of the catatonic digital stationary of a desktop computer is now loose at large, and it becomes intoxicating when it will be shared and digested by the same individuals.

For Lacan the "mirror stage"(Lacan, 2001) is the first step into separating the baby from his mother and introducing him to his reality as a separate entity that is in relation with the others, and thus the discovery of the internal otherness, "The objet (a) is something from 
which the subject, in order to constitute itself, has separated itself off as organ. This serves as a symbol of the lack, that is to say, of the phallus, not as such, but in so far that it is lacking. It must, therefore, be an object that is, firstly, separable and, secondly, that has some relation to the lack." ${ }^{\text {In }}$ the case of mobile connectivity the matter becomes more complicated as the reflected image on the screen in the form of a video "selfie" for instant becomes an image which is not a mere reflection but also image that can be repeated and it can be sent to others that might also reverse this interaction. The direct video communication that is also registered and repeated complicates the matter even further. The "Sardine Can" 4 of Lacan becomes more than just an object that returns the gaze, it become alive and will directly gazes at humans, the image created between the object and the eye is not only mediated so that reality becomes an image but rather the image and reality are mixed together losing each other and lost in each other, there is no more a sharp separation between the image and the actual. The flux is so fast and omnipresent that the boundaries between the two entities of the virtual and the actual are blurry and inconsistent to the extent that the individual user of media augmentation cannot distinguish the difference. Actual reality has exploded in the virtual and more importantly; the latent images of the virtual now exploded in the actual augmenting it to become an actual and physical space of hyperactivity and virtual flux. The end user of this media is now in total control not only of his image but also in control of his actual reality through this direct and remote control of the image. The ability to produce and share an image of the self even when the user is totally immersed in actual reality means that this user has the ability to control and reproduce not only her/his image but also actual physical reality.

The "Selfie" syndrome that is accruing all over social media has the uncanny power of otherness even without the awareness of people producing it. We can see this gaze of otherness in the photographs of David Goldblatt "Ex-Offenders at the Scene of Crime"(Goldblatt, 2011) at the Venice Biennale 2011, with portraits of perpetrators of crimes in their initial location, the gaze in the eyes of the subjects as if coming from beyond, a look of otherness hovering their faces as if they are not them looking at the camera but someone else is behind their eyes returning the gaze to the lens to the photographer to the perceiver to the perpetrator, as if it is this feeling of guilt that haunts them, the other small (a) representing the victims from beyond the grave seeking revenge, the victims possessing the bodies of their perpetrators to pass on their anguish and torment. All the "Selfie" producers have that look of otherness in their eyes as if waiting for eviction, for salvation from the status of the guilt to finally rest in peace, they seem like morbid portraits of the self coming from the beyond, from the realm of the in-between, the "undead" as Jalal Toufic likes to call it, he wrote about this coming back or this call from the dead saying: "to fight the anonymity with which the enemy is killed, the soldier has to receive, from their state of being already dead, the calls of the unknown persons who will soon be murdered by him. Such a call is possible in the non-linear time of undeath".(Toufic, 2014) The gaze of the people of the "Selfie" is that kind of look into the other, even when this other is oneself; it is this small (a) otherness and the feeling of guilt. They are oblivious and confused in the site of their own image on that mesmerizing screen of the phone, oblivious of their own death. The compulsion to repeat, replaces the impulsive to remember, drawing on Freud's "timelessness of the unconscious", and in relation to the "Selfie" phenomenon the original is now and here in hyper time and is hopelessly perishing in an immediate and incessant way in this timelessness of the virtual correlated with actual time, as the subject of the photograph is himself the photographer; the perpetrator of his own death in present time, and his resurrection in hyper time.

Controlled artificial experiences are basically, the result of the human imagination correlated with a generative stimulus like a narrative, a fiction or just any contextual frame to take the user into a created and controlled environment of fantasy. In this constructed environment the actual is suspended and neglected for the purpose of forming a successful mental construct of complete immersion into the imagined or the fantasized. With the advent of new technology of digital communication and the ability to create a virtual reality out of the imagined and the fantasized, people resorted to such environment to live the ultimatum of construct and to suspend actual reality and all its burdens, in the virtual they were able to live a light life of freedom with extraordinary abilities of manipulating and reconstructing their lives within the boundaries of the virtual. But with new technologies of augmentation this latent utopia of freedom and power that was trapped in the virtual has erupted in the actual in a flagrant as a blatant explosion, people felt they have all the capabilities of the virtual reality they have enjoyed for long in the realm of the actual itself, and this burst of digital media in the actual physicality required a new set of technologies that will enable them to implement the way of life of virtuality in the actual. Manipulation of images now has to become manipulation of life itself and thus the creation of an augmented space in the actual becomes a prerequisite.

\section{INSTANTANEOUS, CONTINUOUS AND INTERACTIVE INFORMATION}

With new communication technologies the element of time took a new level of importance, it is not anymore how fast information travels because it is not about the speed anymore since time itself is negated along with instantaneous information. Same information are here and elsewhere at the same time, in no time. Now, real time information is the norm and delay becomes obsolete. In addition to that this information is continuous and available anytime anywhere, non-stop and for free. This kind of information allows the 
interference of the spectator, it is interactive in a sense that it is the product of everybody and available for everyone. Everybody is the producer, the audience and the interpreter of the image; Roland Barthes's "death of the author"'(Barthes, 1978) becomes literally true.

The notion of time is taking more and more importance with any technology that enhances speed, whether it is the speed of transportation in the actual space or speed of information in the virtual realm. The faster people travel, the less time they spend and the more distance they cross. The space-time relation becomes an important variable for existence, history itself is no more a chronological set of narratives but yet a space-time of existence bound to flux and instability. With the industrial revolution exemplified by the invention of the engine and the changes they acquire to the modes of transportation, speed reduced distances, and time diminished space. With the information technology revolution and the invention of yet another engine, specifically the jet engine, it implies a change in the speed of communication and information. The jet engine enables the satellite technology and thus new modes of information transmit around the globe in a live broadcast, the instantaneity of the circulation of information rendered time into zero. In that sense the space-time duality becomes space with no time, the nullification of the notion of time also had its effect on space itself. The instantaneity of transportation is only on the level of non-material information; so physical transportation is still relying on time even when it is reduced and shrunken but it is not yet nullified since there is no teleporting technology yet. This instantaneity is on the level of non-tangible information and thus in a non-tangible space, the negation of time requires, as such, a negation of physical space, and existence becomes non-time / non-space duality, or what is known as the virtual reality that does not abide with the notions of time nor space. Information in that non-space environment is instantaneous since it is also a non-time environment, and it is continuous as long as the physical infrastructure of that virtuality is sustained to feed the tools of connectivity with power and networking. When this technology of immediate information becomes available for the masses with an affordable price, information itself becomes also available and thus very interactive. Not only all information is available for all the people but also those people became the contributors in building a huge archive of information.

The move form actual reality and analogue information to virtual reality and digital information required a separation from the actual space into the no-space; this meant an alienation that the dweller of that space felt. Although the space of the virtual is a space of unlimited capabilities and perfection but this only goes to the nontangible digital construct and thus lacking the human physical presences with most of its senses; in that realm of virtuality people are able to see and to hear but not to touch, to smell or to taste, that meant that humans has to live only partially in that environment waiting for newer technologies to give them full immersion and physical satisfaction. But what happened might be the reverse, with the advent of mobile connectivity technologies people were brought back to the actual tangible space where they can fully engage with all their five senses but equipped with all the technologies of the virtual to be overlaid and juxtaposed with the actual. The shift is thus reversed, starting first from the analogue with industrial technologies of mass production, going to the digital with information technologies of instantaneous production and then back to the actual accompanied by virtuality with the augmentation technologies of mobile connectivity. To the three factors of the virtual, "instantaneous, continuous and interactive" there should be added today another factor, which is "mobile".

Augmented technologies are becoming the norm everywhere, the minimum requirement to create an augmented space are easy to achieve worldwide, even in the most remote countries and even under abnormal conditions of war and instability the creation of an augmented space proved to be easier, and some times more important, than providing drinking water. The mere fact that any wireless internet connection is available make the space an augmented space whether it is $\mathrm{Wi}-\mathrm{Fi}, 3 \mathrm{G}$, or any kind of direct satellite connection which is available without the dependency on any local infrastructure. And this augmented space is a space for violence in many ways depending on the condition of use of it. In Syria, for instances, an augmented space is used in the war effort as a weapon, in fact it became the most influential weapon in that war. Very basic augmented spaces in Syria are created by media activists under heavy circumstances of embargo and shelling to show the images of the atrocity of the war and to create propaganda for the revolution. In any case these augmented spaces that are the backbone of the alternative media are becoming one major weapon that the ruling regime is not able to shut down even after cutting all the basic infrastructure. This media becomes a space of violence in what is presenting and in the way it is used by the revolutionary forces and the regime as a weapon of war and also a space of violence in how much efforts and sacrifices it requires to be created under the difficult situation of war, many activist were killed while installing an internet antenna or while making videos or taking photos, for the Syrian army of the regime a camera is considered more dangerous than a gun.

Augmented space is a space for violence even in the most civilized cities and under the most peaceful conditions as it is a space full of information that circulates between the users of that space, and a space of surveillance and monitoring exercised by the servers operating that space to collect information about the end users that can be utilized in so many ways of advertising and marketing or even for security purposes invading the 
privacy of the individual and affecting the way people conduct their lives to the smallest detail. In Korea, for example, augmented spaces are everywhere in the cities, all sorts of wireless internet connections, giant screens covering the building facades, and small ones on every corner, in the public transportation or shopping malls, surveillance cameras, movement sensors and interactive devises like vending machines or vending walls. An individual is overwhelmed by information even without having any communication device on her/him; no one can escape the exposure to advertising and marketing or monitored positioning. This space is a panoptical space par excellence, a space to monitor and enforce "discipline" in a Foucauldian sense; in short, it is a space 'of violence'. And also a space 'for violence' in so many cases were the spectacle on site is most of the time coined with aggressiveness and action, what is worth showing is a freak show. Media augmentation is banalizing any act of violence and normalizing atrocities and flagrant inhuman acts through transforming them into entertainment shows.

\section{THE PHYSICAL BODY, FROM VIRTUAL AVATARS TO PLASTIC SURGERY}

The advent of image manipulation Softwares into the market in the 90s, namely Photoshop, and its wide accessibility and usability by the public marked an unprecedented hyper-real image in quality and, because of it easiness, a large quantity of images. Photoshop is widely used by the public to aesthetically enhance the image of the human figure. In addition to that, some other Softwares and Applications had the option to automatically modify and enhance the images without even notifying the user, and thus image improvement, like red-eye fixer and bright-skin garnish were becoming normal. This normalized image of the "perfect" human figure led eventually to demand that that image becomes also applicable on the physical body itself, that ultimately led to plastic surgery in actual reality or augmented reality. This heed for the perfect body that originates from the pop image was depicted early in the In 90's by the French artist Orlan as she started a series of chirurgical performances entitled "The Reincarnation of Saint-Orlan"(Orlan, n.d.) where she tried to transform her body into the perfect female body through a set of plastic surgeries that will enact the paintings of the icons of beauty created by major male artists. In that work Orlan is criticizing the standardization of female beauty and how it is manufactured to convey the mainstream image of females through the eyes of mail artists, or in a more contemporary manner, by media gurus, fashion industry and pop stars criteria.

When living in the realm of virtual reality, people give away their physical existence with it their physical body. They learned to be less dependent on their bodily existence and thus they worked on hiding this corporal mass with different interfaces created and generated by computers. The perfect body under virtual reality is a "silent body" it became the Deleuzian body; A body that does not have any physical needs, a "body without organs" or an image of a body that is a manifestation of a deeper reality constructed from fully functioning virtual parts. Every actual body has a virtual dimension, a vast collection of paradigms that constructs its personality, its links, its distresses and its fluxes. This collection of paradigms is what Deleuze calls the "body without organs". In cyber space, people professed the creation of avatars, they mastered regenerating their bodily image with a perfect hyper corporality. The fantasized human in virtual reality is a utopic existence where people are able to fulfil all their caprices and can live life to the extreme; they can satisfy their most intense whims. But eventually this human physiological body is here and now, present and heavy. The physical needs of that body are cumbersome, it ages and gets tired and requires a lot of maintenance. This body "full of organs" is driven by instinctive needs of love and hate, of life and death, of physical needs like eating, sleeping and having sex and thus driven by desires that can never be fulfilled in the virtual.

This transition of the body from the physical to the virtual avatar and back again to its physicality (equipped with electronic devices as cyborg) required a transformation in the means of technologies of communication first and foremost, then it required a transformation in the notion of space and time, public space versus private, and present time versus real time. The preparation of the body for disappearance, for a silent physicality that enables it to enter the realm of the virtual as an image, required a lot of efforts and skills. In that world of images what remains from the tangible body is only what is necessary for its basic survival, and the organs that allowed it to connect to the machine. The body must be maintained to create a spectacle for the screen, powdered and cleansed, ready to be under spotlights, in the shopping windows of the virtual. A body as such, is a body that suggests energy while it is flabby, strength while it is weak, new and unused while it is ravaged by time; a re-delivered baby-body that is hundred years old. This energetic and vigorous body marginalizes few of its irreplaceable senses; it becomes a taste-less and smell-less body and thus outside the realm of conventional space of touch and proximity, into the hyper non-space of the three dimensional illusion of the screen that distances the interlocutors physical bodies apart. In that space the body is guided by different parameters of vectors and digital formulas not anymore by its tangible senses. And thus, throughout the heydays of virtual reality, those actual living bodies are avoided keeping of them, as Bilal Khbeiz suggests: "nothing but their surplus images, well chosen and scanned, images of bodies overflowing with health and channelled with energy (...) This malleability precipitates the migration of the body from the province of magic to the domain of functionality, which is in itself 
a first and irreversible step towards mourning"(Khbeiz, 2003), and towards the death that Ulysses warned his sailors from; a death of the enigmatic physiological body and the birth of an ultimate image-body that suggests and posses an ultimate image of holistic truth "what was, what is and what will be". The truth that has emigrated to a virtual reality of a utopic or, more precisely, teletopic existence is still "lacking in one element, its presence in time and space, its unique existence at the place where it happens to be"(Benjamin and Arendt, 1999) its "Aura" as an actual existence that can never be utopic, on the ground, in the desert of the real. The human body is back again, heavy and cumbrous as it ever was, although categorized into definite provinces and meticulously sculpted into the norms and criteria of ideals. The body is to be maintained by arduous physical training, diet, refinement and a set of varieties of cosmetics to embody ideals. But throughout that process the filter of the culture industry excludes a large range of bodies that are not able to pass that fascist strainer; old and aging bodies will not pass, sick and weak bodies will not pass, the handicapped and the hindered bodies are directly categorized as invalid and incorrect bodies that will eventually fade on the margins of this society. What remains on the façade of the spectacle, on mainstream media is a constant parade of models and paradigmatic perfect bodies that are the most efficacious apparatus for the spectacle. The last resort to contain that body becomes the scalpel; violent acts of surgery to put it back into order. Here comes "plastic surgery" as an act of restraint, a freakish act of control, of persecution that will eventually leave the physiological body with deep scars. Plastic surgery as it is professed toady is becoming an act of mutilation that is disfiguring the human body to confine it into the norms of a fantasized image, an image that was long lived as an avatar, becomes today the norm and the reference for the perfect body.

\section{THE PRODUCTION OF SAMENESS}

What is happening today is a commodification of the human corporality on a large scale, the plastic body is in a that sense the perfect body that everybody sought when assembling an avatar in virtual reality and now reached physiologically with a reality augmented by medical new technologies, and as Adorno and Horkheimer said: "A technological rationale is the rationale of domination itself. It is the coercive nature of society alienated from itself. Automobiles, bombs, and movies keep the whole thing together until their levelling element shows its strength in the very wrong which it furthered. It has made the technology of the culture industry no more than the achievement of standardization and mass production, sacrificing whatever involved a distinction between the logic of the work and that of the social system."5 The augmented hominoid body becomes a norm, and is mass reproduced even before it becomes a cybernetic creature; it is produced under rigorous criteria of aesthetics, a set of standards, measurements and capacities that reconstructs the body to fit it under one of the pre-set categories dictated by the mass culture. The result of this computed type of production is an unprecedented uniformity. Everybody looks like everybody now, in actual reality there is a set of categories that each group of people fits in exactingly; a group that look like a certain pop star for example or another that has the same feature of pinup artist or just following the criteria of beauty set by fashion magazines and TV commercials, "every detail is so firmly stamped with sameness that nothing can appear which is not marked at birth, or does not meet with approval at first sight. And the star performers, whether they produce or reproduce, use this jargon as freely and fluently and with as much gusto as if it were the very language which it silenced long ago. Such is the ideal of what is natural in this field of activity, and its influence becomes all the more powerful, the more technique is perfected and diminishes the tension between the finished product and everyday life. The paradox of this routine, which is essentially travesty, can be detected and is often predominant in everything that the culture industry turns out."(Horkheimer and Adorno, 2007) This capitalist culture industry is still triumphant today with the new technologies of augmentation that are ever in use since the day one of their invention, and sometimes invented only for the market use of augmentation. When Adorno and Horkheimer wrote their critic of enlightenment it was still in its original stage, enlightenment was supposed to be an advance of thought and its objectives were supposed to be aimed at human liberation from the forces of nature to install them as masters of their existence and thus masters of the world, with an abundance of freedom and an abundance of happiness, yet what it delivered is total calamity, and distress. Technological progress of modern science in medicine, in arts and in all sorts of industrial production assured happiness and freedom to humans through reason and promised to unfetter people from the constraints of ignorance and cruelty, from violence and hardship; but it delivered the opposite, instead it helped create a world where people will commit atrocities, and will willingly practice methodical killing, and enthusiastically develop weapons of mass destruction, for Adorno and Horkheimer this is only due to the irrational use of reason. In their critique of modern rationality there is also a critique of pre-modernity and in that sense its legitimate to go in that direction to critique a post-modern technological rational. The failures of modern rationality is regenerating with a new face today in what is supposed to be a post-modern setting, and yet again another time, the technological rationality of the capitalist mode of production failed to fulfil the initial promises of happiness and freedom that it always bragged about. There still is a lack of freedom in today's politics, economy and legal structures that indicate an associated failure in the culture enlightenment. This only implies that something fundamental went wrong in the use of new technologies, or maybe it is better to say abuse of those technologies. Today the production of sameness is more then ever 
locking the world towards another catastrophe of image production were violence persist as the main patent of that post VR image. The same patterns of domination that Adorno and Horkheimer talked about in the 60's are still valid and effective today; the domination of nature by human beings, the domination of nature within human beings, and most importantly, the domination of some human beings by others. Such patterns of domination persist due to an essential factor of the crowed psychology; the irrational fear factor, "Humans believe themselves free of fear when there is no longer anything unknown. This has determined the path of demythologization (...) Enlightenment is mythical fear radicalized".(Horkheimer and Adorno, 2007) In a society that lives an illusion of freedom and where free choice is actually choosing between sameness, and when culture becomes a chase of fame and fortune no matter what the cost; the "other" bares the pains and dreads for that pursuit and is shunted away, exploited, or even violently demolished. The means of destruction are yet technologically advanced and more sophisticated then ever and the exploitation may seem less direct than outright slavery, but domination of humans over other humans remains and is now greater and has global magnitudes. The sole motivation of that practice is again the ever-expanding capitalist economy. Today, fundamental change seems impossible and people are becoming more dedicated to the normalized facts and life conditions of mediocrity.

\section{CONCLUSION}

Media augmentation is by its nature an act of violence, because it is a clash between virtual reality and actual reality and this can be seen on so many level of the production whether direct violence or indirect and symbolic. Although its name suggest augmentation as an addition asset or enhancement of quality but what is actually happening is a violent explosion. This can be obvious when talking about media augmentation in the context of war, and it can be less obvious in so many other contexts were violence was symbolic or latent. The implications of that augmentation is also apparent in the modes of perception of audiences in regard to advertising, to the news or just any simple task of moving around or even on the very personal level of communicating with friends and family. But this explosion did not came from a void, the signs of the fragility of virtual reality where apparent in so many situations, like the event of $9 / 11$ that was considered a symbolic event against the simulation and virtual reality that the western masses were living in, or like the global economical crisis that is considered a crack in the quasi perfect crust of virtuality as the international economy inflated with virtual money transfers and e-commerce. Eventually economical crisis must lead to political crisis, all around the world the civil societies started to express their anger and dissatisfaction with economical situation and the hegemony of the ruling classes. This is yet another symptoms of the collapse of the illusion of well being that the masses lived in the virtual era, in the matrix of a capitalistic pseudo heaven that started to shake under the pressure of social media activism and augmentation.

This explosion of the political was also apparent in the mode of production of the human bodily image, where the fantastic image of the human figure that was created in virtual reality with computer plasticity eventually started to surface in the actual as physiological plastic surgeries. The imagined, malleable, and perfect body of a virtual avatar exploded in actual reality as plastic surgery syndrome that followed all the standards and categories of the perfect and imagined body of virtual utopia, and thus changing the status of activity of the human body that lived the indolence of physicality and the hyperactivity of the mind during the virtual era, but now, with media augmentation, the active physical body regain its presence and exploded on the stage of actual reality ready to perform with all its plasticity as a commoditised cyborg to create the ultimate apparatus of a spectacle.

With media augmentation and its ability to track consumption habits it becomes easy to manipulate the consumers into psychographic commodity fetishism that will tackle their individual taste and aspiration according to pre-collected profiles. It becomes easy, with augmented media, to set a defined and fitting social mask that the target audience longed to. In that sense the media is creating the need and the answer through persuasive appeal and psychological manipulation of human desires and aspirations. In providing a variety of choices the audience will have the feeling of making a decision of choosing not knowing that whatever they chose it will lead to the same result, and thus a system of dumbing down that only serves to normalize the idea of consumption without thinking. Media augmentation gives the feeling of reliability as it is always there ready to answer to any demand and thus one tends to rely on it and trust its information without questioning. The individual consumer equipped with augmentation devises will not feel the need to search or question anything when the world is at her/his fingertip. The surplus of information becomes likes poison, it intoxicate and transforms a scene into an ob-scene, transforms signification into banality and render all images into a pornography-like similes of informing the disenchanted.

This is an image of violence at its best, the sheer quantity of information it provides is an act of bombardment by itself, and this is an image that violates the individual's privacy and private space through manipulation, monitoring and surveillance, through direct and indirect control that will serve in changing consumption habits and political choices. 


\section{REFERENCES}

Barthes, R., 1978. Image-Music-Text. Hill and Wang, New York.

Benjamin, W., Arendt, H., 1999. Illuminations. Pimlico, London.

Dondrue, n.d. Adhd in Adults [WWW Document]. StudyMode.

URL

http://www.studymode.com/essays/Adhd-InAdults-593855.html (accessed 6.24.14).

Goldblatt, D., 2011. Ex-Offenders at the Scene of Crime.

Horkheimer, M., Adorno, T.W., 2007. Dialectic of Enlightenment, 1st ed. Stanford University Press.

Khbeiz, B., 2003. Globalization and the Manufacture of Transient Events. The Lebanese Association for Plastic Arts, Ashkal Alwan, Beirut.

Lacan, J., 2001. Ecrits: A Selection. Routledge, London.

Lacan, J., Miller, J.-A., more, \& 0, 1998. The Seminar of Jacques Lacan: The Four Fundamental Concepts of Psychoanalysis. W. W. Norton \& Company, New York etc.

Mroue, R., 2011. The pixilated revolution.

Orlan, n.d. The Reincarnation of Saint-Orlan.

Poster, M., 2001. Jean Baudrillard: selected writings. Stanford University Press, Stanford.

Toufic, J., 2014. Vampires: An Uneasy Essay on the Undead in Film, 1st edition. ed. Station Hill Press, Barrytown, N.Y. : New York, N.Y.

Virilio, P., 2005. The information bomb. Verso, London; New York.

\footnotetext{
1 (Virilio, 2005) P.63

2 (Poster, 2001) P.213

3 (Lacan et al., 1998) P.97

4 (Lacan et al., 1998) P. 95

5 (Horkheimer and Adorno, 2007) p. 95
} 